Púb, Mat. UAB

$N^{\circ} 210 \mathrm{Ct}, 1980$

Actes VII JMHL

FUNCIONES ARITMETICAS DE TIPO MANGOLDT $\Lambda_{\mathrm{k}}^{*} \Lambda_{\mathrm{k}, \mathrm{t}}^{*}$

Catalina Calderón García

Dpto. de Matematicas

Universidad del Pais vasco

ABSTRACT.- From a function of type Möbius, $\mu_{k}$, it is defined ano ther two functions $\Lambda_{k}^{*}$ and $\Lambda_{k}^{k}, t$. Them, it is obtained, as a particular case, the Mangoldt's function $A$.

Para cada $k$ entero positivo definimos la función aritmética $A_{k}^{*}$ por

$$
A_{k}^{*}(n)=\sum_{d / n} \mu_{k}^{*}(d) \log n / d
$$

donde $\mu_{\mathrm{k}}^{*}$ es una función generalizada de Möbius que se define de la siguiente forma:

$$
\begin{aligned}
& \mu \stackrel{k}{k}(1)=1 \\
& \mu_{k}^{*}(n)=0 \quad \text { si } p^{k+1 / n} \text { para algín } p \text { primo } \\
& \mu_{k}^{*}(n)=(-1)^{\Omega(n)} \text { si } n=\prod_{i=1}^{r} p_{i}^{\alpha}, 0 \leq \alpha_{i} \leq k \text {, } \\
& \Omega(n)=\sum_{i=1}^{r} \alpha_{i}
\end{aligned}
$$

Si $k=1$ la función $\mu_{k}^{*}$ coincide con la función de Möbius usual y por lo tanto la formula anterior (1) da la funcion de Mangoldt. 
La serie de Dirichlet que genera la funcion $\Lambda_{k}^{*}$ se obtiene a partir de la correspondiente a la funcion $\mu_{k}^{*}$. Esto es, para la funcion $\mu_{k}^{\star}$ se verifica

$$
\sum_{n=1}^{\infty} \mu_{k}^{*}(n) n^{-s}=\frac{\zeta(2 s)}{\zeta(s)} \gamma_{k}(s), \quad \text { Re } s>1
$$

donde

(4)

$$
\gamma_{k}(s)= \begin{cases}\frac{\zeta((k+1) s)}{\zeta(2(k+1) s)} & \text { si } k \text { es par } \\ \frac{1}{\zeta((k+1) s)} & \text { si } k \text { es impar }\end{cases}
$$

de (3) y (4) y diferenciando termino a termino la función zeta se obtiene en el semiplano $\operatorname{Re} s>1$

$$
\sum_{n=1}^{\infty} \mu_{k}^{\star}(n) \cdot n^{-s} \sum_{n=1}^{\infty} \log n \cdot n^{-s}=\sum_{n=1}^{\infty} A_{k}^{*}(n) \cdot n^{-s}
$$

podemos escribir para A

$$
\sum_{n=1}^{\infty} f_{k}^{*}(n) \cdot n^{-s}=-\frac{\zeta^{*}(s)}{\zeta(s)} \zeta(2 s) Y_{k}(s) \quad \text { Re } s>1
$$

donde $Y_{k}(5)$ está dada por (4).

Se observa en (5) que cuando $k=1$ se obtiene la serie correspondiente a la funcion de Mangoldt usual.

Si $k=2$ la funcion $A_{2}^{\star} \quad y$ la funcion $B$ definida para $n=p_{1}{ }^{\alpha} \ldots p_{r}^{\alpha}$ por $\beta(n)=\alpha_{1} \ldots \alpha_{r}$ siendo los $\alpha_{i}>0$ y $p_{i}$ primos distintos se verifica una propiedad que relaciona estas funciones con la función $\mu$ de Möbius

$$
A_{2}^{*}(n)=-\sum_{d / n} \mu(d) \log d B(n / d)
$$


Una generalización inmediata de la funcion $h_{k}^{\star}$ es la siguiente. Para $k, t$ números naturales cualesquiera definimos la función $\Lambda_{k, t}^{*}$ por la expresión

$$
n_{k, t}^{*}(n)=\sum_{d / n} \mu_{k}^{*}(d)(\log n / d)^{t}
$$

Si $t=1$ obtenemos la funcion (1), $\hat{A}_{k, 1}^{*}(n)=\Lambda_{k}^{*}(n)$.

La diferenciación termino a termino de la función zeta nos da

$$
\zeta^{(t)}(s)=(-1)^{t} \sum_{n=1}^{\infty} \log ^{t} n \cdot n^{-s}
$$

đé (3), (4) y (8) vemos que

$$
\sum_{n=1}^{\infty} \Lambda_{k, t}^{*}(n) \cdot n^{-s}=\sum_{n=1}^{\infty} H_{k}^{*}(n) \cdot n^{-s} \cdot \sum_{n=1}^{\infty} \log ^{t} n \cdot n^{-s}
$$

Ass obtenemos para la funcion $n_{k, t}^{*}$

$$
\sum_{n=1}^{\infty} n_{k, t}^{k}(n) \cdot n^{-s}=(-1)^{t} \frac{\zeta^{(t)}(s)}{\zeta(s)} \zeta(2 s) \gamma_{k}(s) \quad \text { Re } s>1
$$

donde $Y_{k}(s)$ viene dada por (4).

IVIC, A. $|4|$, define una función generalizada de Mangoldt $A_{t}$ de la siguiente forma

$$
n_{t}(n)=\sum_{d / n} \mu(d)(\log n / d)^{t}
$$

y obtiene la serie de Dirichlet para esta funcion, esto es

$$
\sum_{n=1}^{\infty} A_{t}(n) \cdot n^{-s}=(-1)^{t} \frac{\zeta^{(t)}(s)}{\zeta(s)}
$$

Obtendremos a continuación una expresión que relaciona la funcion $\Lambda_{k, t}^{*}$ con la funcion $\Lambda_{k}^{*} y \Lambda_{t}$. 


$$
\frac{1}{\zeta(s)}(-1)^{t} \frac{\zeta^{(t)}(s)}{\zeta(s)} \zeta(2 s) \gamma_{k}(s)=(-1)^{t} \frac{\zeta^{(t)}(s)}{\zeta(s)} \frac{\zeta(2 s)}{\zeta(s)} Y_{k}(s)
$$

se deduce la relacion

$$
\sum_{d / n} \Lambda_{k, t}^{*}(d) \mu(n / d)=\sum_{d / n} \Lambda_{t}(d) H_{k}^{*}(n / d)
$$

Aplicando ahora la inversión de Möbius a la funcion

$$
f(n)=\sum_{d / n} \mu(d) A_{k, t}^{\star}(n / d)
$$

obtenemos por f́ltimo que

$$
\Lambda_{k, t}^{*}(n)=\sum_{d / n} \sum_{m / d} \Lambda_{t}(m) \mu_{k}^{*}(a / m)
$$

\section{BIBLIOGRAF IA}

I1 CALderoN G., C.: "Sobre unas funciones generalizadas de Möbius y Euler". VI Jornadas de Matematicas HispanoLusas. Santander, 1979.

|2| CALderon G., C.: "Algunas propiedades de las funciones $\mu_{k}^{*}$, $\phi_{k}^{\star} "$. Revista de la Academia de Ciencias de Zaragoza.

|3| CHANDRASEKHARAN, K.: "Arithmetical Functions". Springer-Ver lag, Berlin-Heidelberg-New York, 1970.

|4| IVIC, A.: "An Application of Dirichlet series to Certain Arithmetical Functions". Mathematika Balkanica, 3, 158-165, Belgrado, 1973.

|5| PRACHAR, K.: "Prinzahlverteilung". Springer-Verlag, BerlinHei.delberg-New York, 1957. 